Forum 2018 · 33:451

https://doi.org/10.1007/s12312-018-0520-2

Online publiziert: 14. November 2018

C) Springer Medizin Verlag $\mathrm{GmbH}$, ein Teil von Springer Nature 2018

KREBSGESELLSCHAFT

RHEINLAND-PFALZ E.V.
Krebsgesellschaft Rheinland-Pfalz e.V.

\section{Dem Leben eine neue Perspektive geben}

\section{Jahre ambulante psychoonkologische Versorgung in Rheinland-Pfalz}

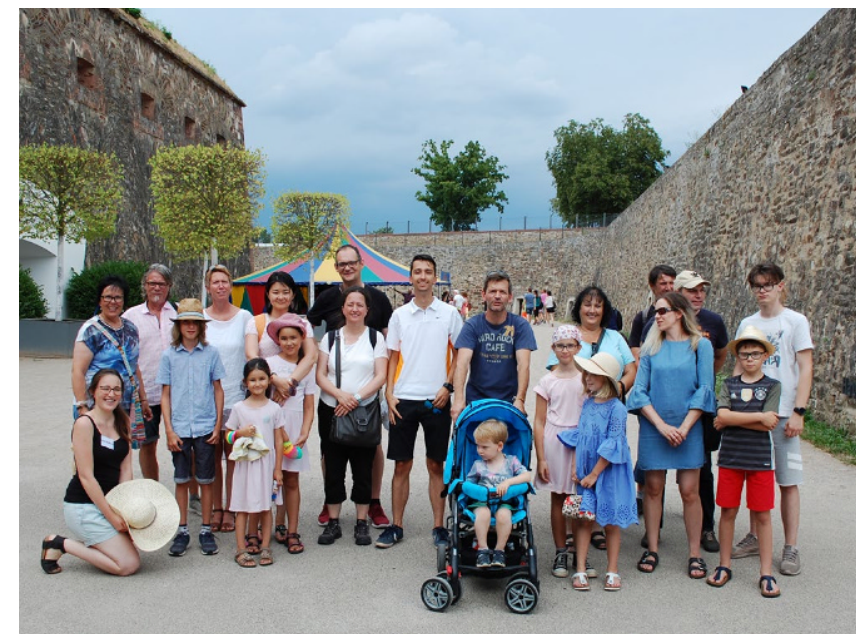

\author{
4 Neun Familien \\ des landesweiten \\ Projektes „Mama/ \\ Papa hat Krebs" der \\ Krebsgesellschaft \\ Rheinland-Pfalz e.V. \\ haben dank der \\ Unterstützung durch \\ das Café Hahn und \\ die Seilbahn Koblenz \\ im Juli 2018 das \\ Gauklerfest auf der \\ Festung Ehren- \\ breitstein besucht. \\ (๑ Krebsgesellschaft \\ Rheinland-Pfalz)
} den, wenn die Seele krank ist." So bot er den Patienten seines Radiologischen Institutes bereits 1974 psychosoziale Beratung und Begleitung in Zusammenarbeit mit der Krebsgesellschaft an. Daraus entwickelte sich nur vier Jahre später eine eigenständige Beratungsstelle mit je einer Beratungs- und einer Verwaltungskraft, die im ersten Jahr ihres Bestehens bereits 800 Beratungskontakte registrieren konnte.

\section{》) Der Leidende kann nicht gesunden, wenn die Seele krank ist}

Inzwischen sind nicht nur das Koblenzer Team und das Angebotsspektrum deutlich gewachsen, sondern auch drei weitere, Beratungszentren in Trier (1983), Ludwigshafen (1986) und Kaiserslautern (1996) sowie zahlreiche temporär besetzte regionale Außenstellen in den jeweiligen Einzugsgebieten hinzugekommen. Pro Jahr verzeichnet die Krebsgesellschaft mittlerweile im Schnitt rund 17.000 Beratungskontakte. Davon entfallen gut zwei Drittel auf überwiegend persönliche, aber auch telefonische und schriftliche Kontakte mit Betroffenen.

Ergänzend zur psychosozialen Beratung runden vielfältige Gruppen, Kurse, Vorträge und Informationsveranstaltungen rund um das Thema Krebs und die Unterstützungsmöglichkeiten zur Bewältigung der Erkrankung das aktuelle Leistungsspektrum ab.

Auch Angehörige sind durch die Erkrankung eines Familienmitgliedes stark belastet und nehmen das Beratungs- und Unterstützungsleistungenangebot in Anspruch. Hier wurden in den letzten Jahren verstärkt die Belange von Kindern und Jugendlichen in den Blick genommen, deren gewohnter Alltag durch die Erkrankung eines Elternteils aus den Fugen geraten ist. Ihre Not wird leicht übersehen, da sie eher dazu neigen, zu verstummen, sich anzupassen oder in anderer Weise auffallig zu werden. Im Rahmen des mittlerweile landesweit angebotenen Projektes „Mama/ Papa hat Krebs" unterstützen die BeraterInnen der Krebsgesellschaft Eltern dabei, altersgerecht und offen mit ihren Kindern über die Erkrankung und mögliche Folgen $\mathrm{zu}$ sprechen. Kinder und Jugendliche haben die Möglichkeit, sich bei Bedarf ihre eigenen Sorgen und Nöte von der Seele zu reden, mit denen sie ihre Eltern nicht noch zusätzlich belasten möchten.

Ein zentraler Baustein des Projektes sind darüber hinaus regelmäßige Gruppenaktivitäten und Familienaktionen, bei denen die Erkrankung vorübergehend in den Hintergrund treten darf und Kraft für den Alltag getankt werden kann. Ermöglicht werden diese Auszeiten dank des Engagements von Förderern, die dafür Spenden oder Sachleistungen wie Freikarten etc. zur Verfügung stellen.

\section{Korrespondenzadresse}

Presse- und Öffentlichkeitsarbeit

Krebsgesellschaft Rheinland-Pfalz e.V.

Löhrstraße 119, 56068 Koblenz, Deutschland cehricht@krebsgesellschaft-rlp.de

\section{Christina Ehricht}

